\title{
Intelligent Controllers for Multirobot Competitive and Dynamic Tracking
}

\author{
Mei Liu, ${ }^{1,2,3}$ Shuai Li $\left(\mathbb{D},{ }^{4}\right.$ Xiaodi Li, ${ }^{5}$ Long Jin $\left(\mathbb{D},{ }^{1,2,3}\right.$ Chenfu Yi, ${ }^{6}$ and Zhiguan Huang ${ }^{1}{ }^{1}$ \\ ${ }^{1}$ Guangdong Provincial Engineering Technology Research Center for Sports Assistive Devices, Guangzhou Sport University, \\ Guangzhou 510000, China \\ ${ }^{2}$ Key Laboratory of IoT of Qinghai Province, Qinghai Normal University, Xining, China \\ ${ }^{3}$ School of Information Science and Engineering, Lanzhou University, China \\ ${ }^{4}$ Department of Computing, The Hong Kong Polytechnic University, Hung Hom, Kowloon, Hong Kong \\ ${ }^{5}$ School of Mathematical Sciences, Shandong Normal University, Jinan, Shandong, China \\ ${ }^{6}$ School of Information Engineering, Jiangxi University of Science and Technology, Ganzhou 341000, China
}

Correspondence should be addressed to Shuai Li; shuaili@polyu.edu.hk, Long Jin; jinlongsysu@foxmail.com, and Zhiguan Huang; zhiguan1980@163.com

Received 1 August 2018; Accepted 2 October 2018; Published 21 October 2018

Guest Editor: Andy Annamalai

Copyright ( $\odot 2018$ Mei Liu et al. This is an open access article distributed under the Creative Commons Attribution License, which permits unrestricted use, distribution, and reproduction in any medium, provided the original work is properly cited.

\begin{abstract}
This paper focuses on the problem of target tracking using $k$ fittest robots in a group of $n$ mobile robots with $n>k$. We present centralized and distributed coordination models with all-to-all and limited communications, respectively. For the case of all-to-all communication between robots, theoretical analysis is presented to prove the exponential stability of the whole system. In real applications of robotic networks, a robot may only be allowed to exchange information with a limited number of neighbors. In such a limited communication case where centralized quantity is not available, a consensus filter is used to estimate the centralized quantities in a distributed way, and a distributed competitive target tracking is thus achieved. The stability of the distributed control is also proved in theory. Finally, illustrative examples are provided and analyzed to substantiate the efficacy of the proposed models for tracking moving target in a competition manner with all-to-all communications and limited communications.
\end{abstract}

\section{Introduction}

The competition and cooperation behaviors of living beings, such as swarms of ants, shoals of fish, or even the troops in military, have certain advantages, including avoiding predators, increasing the chance of finding food, saving energy, and so on. For example, for a joint vigilance task in military, all soldiers should keep unmoved and the one who finds an invader should track the invader closely. Such a behavior can be deemed as a coordination based on the competition, where the soldier who finds the invader is the "winner" and wins the opportunity to do the tracking task while the rest ones are the "losers" and keep unmoved to do the vigilance.

Deemed as a modeling of cooperation, consensus algorithms estimate the related weights or states by mitigating differences among multiple agents in a group and have been applied to many computation problems depicted in distributed manner, such as multiagent systems [1-3], consensus for multiple robots [4], distributed environmental modeling [5]. Despite the achieved success, consensus essentially lacks a mechanism to model competition behaviors in a distributed network, which desires the increase of peer differences and the enhancement of contrasts [6].

Research in many fields observes and confirms the fact that the competition is equally important as the cooperation for complex systems [6-8]. Therefore, various schemes and models have been proposed, investigated, and applied to describe and model the competition. Among them, the $k$-winners-take-all ( $k$-WTA) models, which identify the $k$ largest of $n$ competitors with $n>k$, are a typical approach to capture competitive behaviors. In addition, the $k$-WTA degrades to the famous winner-take-all (WTA) process for $k=1$ [9-13]. It is proven in [9] that a two-layered network aided with WTA is able to approximate any nonlinear 
mapping in arbitrary accuracy. In addition, it is proven in [11] that a $k$-WTA problem can be equivalently converted to a constrained convex quadratic programming (QP) optimization formulation, which enriches significantly techniques for solving $k$-WTA problems. Thanks to the advantages of intelligent computing and universal approximation, neural networks have been widely investigated in numerous areas [14-20]. For example, an adaptive control approach based on neural network is constructed to stabilize a class of nonlinear discrete-time systems with unknown functions and unknown control direction, in which the neural networks are exploited to approximate the unknown function [18]. Being designed for solving QP problems, dual neural networks are constructed in [16] and applied to solving the redundancy resolution of kinematically redundant manipulators, which utilizes the corresponding dual variables. Dual neural networks are then further exploited to solving $k$-WTA problems in $[10,12,13]$. The $k$-WTA problem is modeled as a convex QP problem and then solved by an elaborately constructed dual neural network in [10]. By following the same problem formulation, the model in [10] is simplified and modified in [12] by tailoring the structure and taking advantage of the nonlinearity provided by a saturation function used in the model.

Currently, robotics is playing an indispensable role in modern society as well as academic researches and industrial applications [21-34]. Yang et al. introduce physical haptic feedback mechanism in robots to result in muscle activity, in order to achieve intuitive human impedance transfer through a designed coupling interface in [21]. In addition, multiple mobile robots coordination plays an important role in various fields such as cooperative rescue, antiterrorism and is becoming a hot but tough topic in robotics [23, 29, 31, 35]. For example, the internal forces applied on the object for a dual-arm robot are considered in addition to the external forces in [23]. The coordination of multiple fixed-base redundant manipulators are investigated in [8] to execute a given task with dynamic task allocation ability. A multiplerobot system can be deemed as a robot group in which the robots can communicate with others and can cooperatively implement different kinds of tasks. A near-optimal tracking control method is presented for wheeled mobile robots in [29], which designs a backstepping kinematic controller to generate desired velocity profiles. The moving target tracking with a group of mobile robots is investigated in [31], which allows each robot in the group has a pan/tilt camera to detect the target and has limited communication capability of communicating with neighbor robots. This is close to what we are investigating but with a major difference that they drive all the mobile robots to track the moving target while only the winners of the mobile robots in this paper can be activated to do the task.

Although some achievements have been earned for the cooperative control of multiple mobile robots, there are still some unsatisfactory aspects with existing solutions. One of the unsatisfactory aspects in the existing achievements is that they can not select the fittest mobile robots in a group to execute the task with the rest ones unmoved. In this paper, we use multiple mobile robots for tracking target in a competition manner, which extends $k$-WTA to the study of complex behavior coordination in a swarm of robots. We first propose a target tracking model with all-to-all communications. Then, a modified model only requiring limited communications among mobile robots is proposed to solve this problem. Theoretical analyses are provided to prove the exponential stability. Both models are validated by computer simulations.

The remainder of this paper is organized into five sections. The preliminary for tracking target via multiple mobile robots in a competition manner is presented in Section 2, which is consisted of the modeling on the mobile robot, the assumptions as well as the theoretical basis for analyzing the proposed models. Then, the coordination model for tracking the moving target with all-to-all communications is designed and analyzed in Section 3. Section 4 uses a distributed consensus filter to estimate centralized quantities, and then embeds it into the distributed coordination based model with limited communications with theoretical analyses on convergence speed and stability presented. Section 5 provides illustrative simulation examples to substantiate the efficacy and superiority of the proposed models for tracking target via multiple mobile robots in the situations of all-to-all communications and limited communications. Section 6 concludes the paper with final remarks.

\section{Preliminary and Problem Formulation}

This section presents the preliminary and the model of the robot.

2.1. Preliminary. In the ensuing sections, the coordination models for multiple mobile robots will be viewed as singularly perturbed systems. Therefore, for investigating the convergence speed and stability of these models, the following theoretical basis on a singularly perturbed system is presented as a preliminary, which will be utilized in the ensuing proofs of Theorems 3 and 4 .

Lemma 1 (see [36]). Consider the following singularly perturbed system:

$$
\begin{aligned}
\dot{\omega} & =\mu(\omega, \varrho, \epsilon), \\
\epsilon \dot{\varrho} & =\vartheta(\omega, \varrho, \epsilon),
\end{aligned}
$$

where $\omega \in \mathbb{R}^{v}$ and $\varrho(t) \in \mathbb{R}^{\chi}$ denote state vectors of the system and $\epsilon>0 \in \mathbb{R}$ is a constant parameter; functions $\mu: \mathbb{R}^{\nu+\chi+1} \longrightarrow \mathbb{R}^{\nu}, \vartheta: \mathbb{R}^{\nu+\chi+1} \longrightarrow \mathbb{R}^{\chi}$ are smooth.

Assume that the following conditions are satisfied for all $(\omega, \epsilon) \in B_{v} \times\left[0, \epsilon_{0}\right]$.

(1) $\mu(0,0, \epsilon)=0$ and $\vartheta(0,0, \epsilon)=0$.

(2) The equation $\vartheta(\omega, \varrho, 0)=0$ has an isolated root $\varrho=$ $\phi(\omega)$ such that $\phi(\Phi)=0$, where $\phi: \mathbb{R}^{v} \longrightarrow \mathbb{R}^{\chi}$ is a smooth function.

(3) The functions $\mu, \vartheta, \phi$ and their partial derivatives up to the second order are bounded for $\varrho-\phi(\omega) \in B_{\chi}$. 
(4) The origin of the reduced system $\dot{\omega}=\mu(\omega, \phi(\omega), 0)$ is exponentially stable.

(5) The origin of the bounded-layer system

$$
\frac{\mathrm{d} \omega}{\mathrm{d} \widehat{\tau}}=\vartheta(\omega, \omega+\phi(\omega), 0)
$$

is exponentially stable, where $\omega=\varrho-\phi(\omega)$ and $\widehat{\tau}=t / \epsilon$. Then, there exists $\epsilon^{*}>0 \in \mathbb{R}$ such that, for all $\epsilon<\epsilon^{*}$, the origin $(\omega=0, \varrho=0)$ of system (1) is exponentially stable.

2.2. Mathematical Symbols and Meanings. To lay a basis for further investigation, the mathematical symbols and their meanings used in this paper are listed as follows:

$\left(x_{i}, y_{i}\right)$ : Cartesian coordinates of the middle point of the driving wheel axle of the $i$ th mobile robot.

$\alpha_{i}$ : bearing of the robot platform with respect to the $\mathrm{x}$-axis of the $i$ th mobile robot.

$l$ : length between the two driving wheels.

$\xi_{i 1}$ : speed of the left wheel of the $i$ th mobile robot.

$\xi_{i r}$ : speed of the right wheel of the $i$ th mobile robot.

$c c>0$.

$p_{i} \in \mathbb{R}^{m}$ : position of the $i$ th mobile robot.

$u_{i} \in \mathbb{R}^{m}$ : control input (the velocity) of the $i$ th mobile robot.

$z \in \mathbb{R}$ : auxiliary variable and can be initialized randomly.

$\rho \rho>0$.

$p_{\mathrm{t}}$ : position of the moving target.

$\dot{p}_{\mathrm{t}}$ : velocity of the moving target.

$\lambda$ : feedback gain.

$\otimes$ : the Kronecker product.

$I_{2 n}$ : vector composed of $2 n$ elements with each one being 1 .

$I_{n}$ : vector composed of $n$ elements with each one being 1 .

$I_{2}$ : vector composed of 2 elements with each one being 1 .

2.3. Differential-Driven Robot. The differential-driven-wheeled mobile robot (Figure 1) is equipped with two step motors for movement, Bluetooth module for communication, and an on-board microprocessor for programming and control. The kinematic model of the $i$ th differential-driven-wheeled mobile robot is formulated as

$$
\left[\begin{array}{c}
\dot{x}_{i} \\
\dot{y}_{i} \\
\dot{\alpha}_{i}
\end{array}\right]=\left[\begin{array}{cc}
\frac{\cos \alpha_{i}}{2} & \frac{\cos \alpha_{i}}{2} \\
\frac{\sin \alpha_{i}}{2} & \frac{\sin \alpha_{i}}{2} \\
\frac{-1}{l} & \frac{1}{l}
\end{array}\right]\left[\begin{array}{l}
\xi_{i 1} \\
\xi_{i 2}
\end{array}\right] .
$$

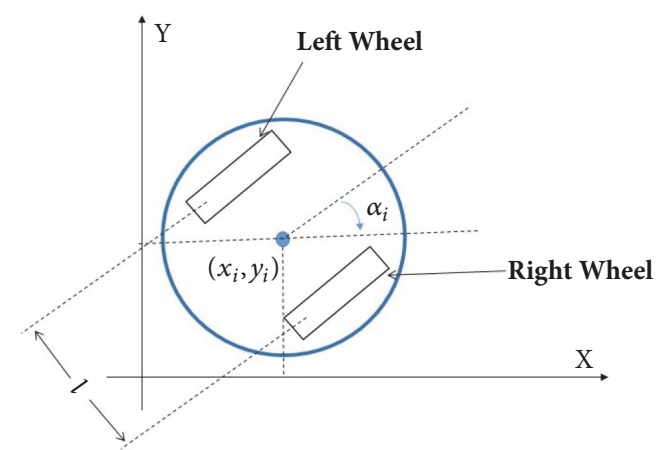

FIGURE 1: Differential-driven-wheeled mobile robot model.

With the aid of feedback linearization technique presented in [37], we have

$$
\begin{aligned}
& {\left[\begin{array}{l}
\xi_{i 1} \\
\xi_{i 2}
\end{array}\right]} \\
& \quad=\left[\begin{array}{cc}
\frac{l \sin \alpha_{i}}{2 c}+\cos \alpha_{i} & \frac{-l \cos \alpha_{i}}{2 c}+\sin \alpha_{i} \\
\frac{-l \sin \alpha_{i}}{2 c}+\cos \alpha_{i} & \frac{l \cos \alpha_{i}}{2 c}+\sin \alpha_{i}
\end{array}\right]\left[\begin{array}{c}
\dot{p}_{i 1} \\
\dot{p}_{i 2}
\end{array}\right] .
\end{aligned}
$$

The motion of the $i$ th robot is

$$
\dot{p}_{i}=u_{i}
$$

2.4. Problem Definitions. In this part, problem definitions are presented as follows.

Problem 1 (centralized competitive tracking). With all-toall communications, construct a model for $n$ mobile robots described by (5) to allocate the task to the fittest robots such that the $k$ mobile robots with the minimum distance from the moving target stay active to execute the task while the others keep unmoved.

Problem 2 (distributed competitive tracking). With limited communications, design a model to achieve the same goal as presented in Problem 1.

\section{Competitive Tracking with All-to-All Communications}

This section presents a centralized competition control law for tracking the moving target with all-to-all communications.

3.1. Model Design. The neural network model presented in [12] for generating $k$-WTA competition is formulated as

$$
\begin{gathered}
\frac{\mathrm{d} z}{\mathrm{~d} t}=-\rho\left(\sum_{i=1}^{n} \delta_{i}-k\right) \\
\delta_{i}=\sigma_{\Omega i}\left(z+\frac{v_{i}}{2 a}\right),
\end{gathered}
$$




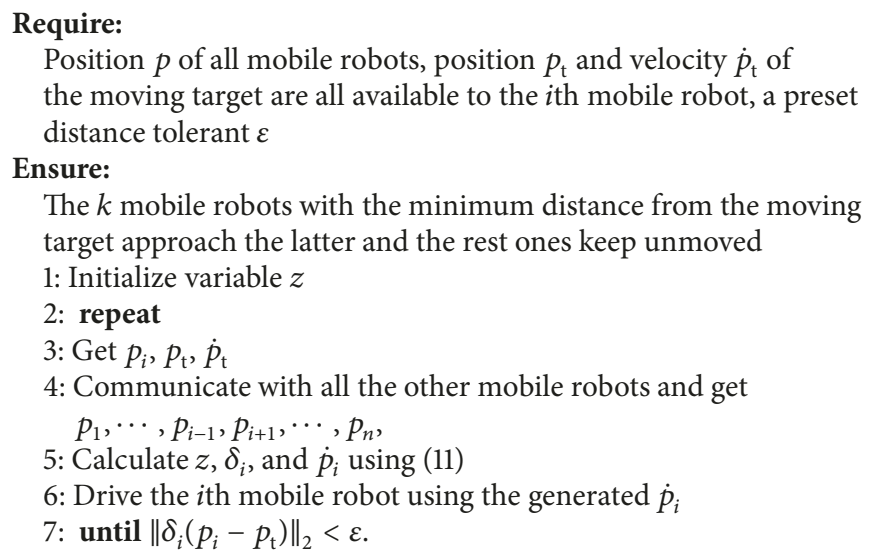

Algorithm 1: Centralized competitive target tracking control for the $i$ th mobile robot with all-to-all communication.

where $v_{i}$ is the $i$ th element of $v ; \delta_{i}$ stands for the $i$ th element of $\delta \in\{0,1\}^{n} ; a>0$ satisfies $a \leq 0.5\left(\bar{v}_{k}-\bar{v}_{k+1}\right) ; \sigma_{\Omega i}(\cdot)$ is defined as

$$
\sigma_{\Omega i}\left(z+\frac{v_{i}}{2 a}\right)= \begin{cases}1, & \text { if } z+\frac{v_{i}}{2 a}>1 \\ z+\frac{v_{i}}{2 a}, & \text { if } 0 \leq z+\frac{v_{i}}{2 a} \leq 1 \\ 0, & \text { if } z+\frac{v_{i}}{2 a}<0 .\end{cases}
$$

The above neural network model is globally asymptotically stable for solving the $k$-WTA problem [12]:

$$
\delta_{i}=f\left(v_{i}\right)= \begin{cases}1, & \text { if } v_{i} \in\{k \text { largest elements of } v\} \\ 0, & \text { otherwise. }\end{cases}
$$

In addition, the control for the $i$ th mobile robot is defined as

$$
\dot{p}_{i}=-\delta_{i}\left(\lambda\left(p_{i}-p_{\mathrm{t}}\right)+\dot{p}_{\mathrm{t}}\right) \text {. }
$$

It can be obtained from (10) that the $i$ th mobile robot is unmoved for $\delta_{i}=0$ and that the $i$ th mobile robot approaches to the moving target exponentially for $\delta_{i}=1$.

Substituting (7) into (6) generates

$$
\begin{aligned}
& \dot{p}_{i}=-\sigma_{\Omega i}\left(z+\frac{v_{i}}{2 a}\right)\left(\lambda\left(p_{i}-p_{\mathrm{t}}\right)+\dot{p}_{\mathrm{t}}\right), \\
& \dot{z}=-\rho\left\{\sum_{i=1}^{n} \sigma_{\Omega i}\left(z+\frac{v_{i}}{2 a}\right)-k\right\},
\end{aligned}
$$

where $v_{i}=-\left\|p_{i}-p_{\mathrm{t}}\right\|_{2}$. In addition, the coordination model for tracking the moving target can be formulated into a compact form:

$$
\begin{aligned}
& \dot{p} \\
& \quad=-\sigma_{\Omega}\left(z I_{2 n}+\frac{v}{2 a} \otimes I_{2}\right)\left(\lambda\left(p-I_{n} \otimes p_{\mathrm{t}}\right)+I_{n} \otimes \dot{p}_{\mathrm{t}}\right), \\
& \dot{z}=-\rho\left(I_{n}^{\mathrm{T}} \sigma_{\Omega}\left(z I_{n}+\frac{v}{2 a}\right)-k\right) .
\end{aligned}
$$

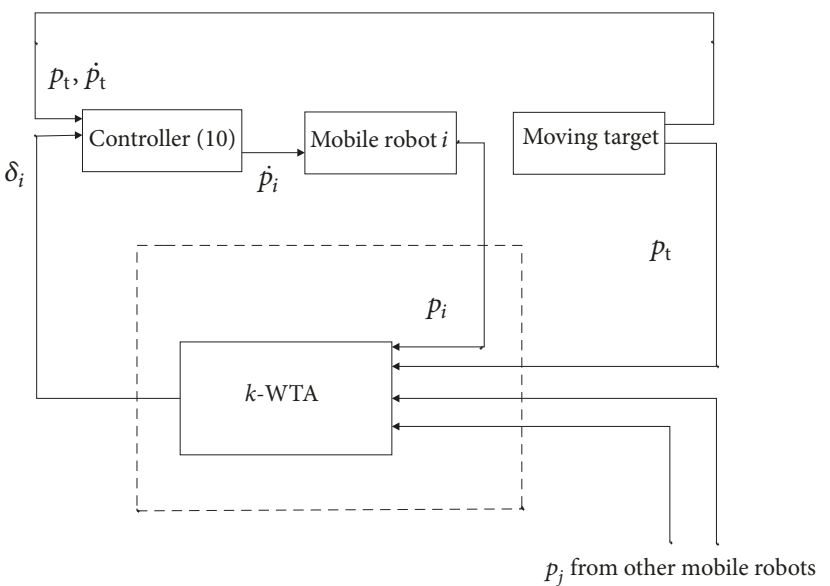

FIGURE 2: Control block diagram for the ith mobile robot in the centralized competitive target tracking control via multiple robots in the situation of all-to-all communications, where subscript $j \in$ $\{1, \ldots, i-1, i+1, \ldots, n\}$.

As illustrated in Figure 2, the proposed centralized coordination model consists of two parts: the $k$-WTA part (as shown in the dashed rectangle in the figure) and the controller part. The $k$-WTA part collects inputs from the robots (e.g., $p_{i}$ ), and inputs from the moving target (i.e., $p_{\mathrm{t}}$ ) and outputs $\delta_{i}$, which is feeded into the controller (10). Then, the control input $\dot{p}_{i}$ to the mobile robot is yielded. The diagram illustrates the interplay between the mobile robot, the moving target and the coordination model. In addition, procedures of the proposed centralized coordination model with all-to-all communications are stated in Algorithm 1 . The $i$ th mobile robot first collects its position $p_{i}$, the position $p_{\mathrm{t}}$ and velocity $\dot{p}_{\mathrm{t}}$ of the moving target (Line 3 ), and collects position of all the other robots by communication (Line 4). After this, $\dot{p}_{i}$ is calculated using (11) and then drive the mobile robot using $\dot{p}_{i}$. Lines $3,4,5$, and 6 are repeated in sequence until the preset distance tolerance is achieved. 
In addition, we have the following theorem for the proposed centralized coordination model (12) for competitive tracking.

Theorem 3. There exists $\rho^{*}>0 \in \mathbb{R}$ such that, for all $\rho>\rho^{*}$, for a group of $n$ differential-driven robots described by (5) with the coordination control law (12), $k$ robots with the minimum distance move towards the target at an exponential speed.

Proof. The proof involves two aspects. (1) The equilibrium point of system (12) is identical to the solution of Problem 1. (2) System (12) exponentially converges to its equilibrium point.

For aspect (1), it is evident that the equilibrium point of system (12) corresponds to the $k$ mobile robots with minimum initial distance from the moving target capturing the latter. That is, the equilibrium point of system (12) is identical to the solution of Problem 1.

For aspect (2), the centralized coordination model (12) can be rewritten as

$$
\begin{aligned}
p & =\mu(p, z, \rho) \\
& =-\sigma_{\Omega}\left(z I_{2 n}+\frac{v}{2 a} \otimes I_{2}\right)\left(\lambda\left(p-I_{n} \otimes p_{\mathrm{t}}\right)+I_{n} \otimes \dot{p}_{\mathrm{t}}\right) \\
\frac{1}{\rho} \dot{z} & =\vartheta(p, z, \rho)=-\left(I_{n}^{\mathrm{T}} \sigma_{\Omega}\left(z I_{n}+\frac{v}{2 a}\right)-k\right),
\end{aligned}
$$

of which the equilibrium point is $\left(p^{*}, z^{*}\right)$. Evidently, the above system is a special case of singularly perturbed system (1). Therefore, the stability of the equilibrium point is evaluated via Lemma 1.

Using the linear translation $p^{\prime}=p-p^{*}$ and $z^{\prime}=z-z^{*}$, we have $p=p^{\prime}+p^{*}$ and $z=z^{\prime}+z^{*}$, and further have

$$
\begin{aligned}
\dot{p}^{\prime} & =\mu\left(p^{\prime}+p^{*}, z^{\prime}+z^{*}, \rho\right), \\
\frac{1}{\rho} \dot{z}^{\prime} & =\vartheta\left(p^{\prime}+p^{*}, z^{\prime}+z^{*}, \rho\right) .
\end{aligned}
$$

It can be further derived that

$$
\begin{aligned}
\dot{p}^{\prime} & =\mu\left(p^{*}, z^{*}, \rho\right)=0, \\
\frac{1}{\rho} \dot{z^{\prime}} & =\vartheta\left(p^{*}, z^{*}, \rho\right)=0,
\end{aligned}
$$

where $p^{\prime}=z^{\prime}=0$ are the new equilibrium points. Therefore, condition (1) is satisfied. It can be generalized from Corollary 1 in [12] that $I_{n}^{\mathrm{T}} \sigma_{\Omega}\left(z I_{n}+v / 2 a\right)-k=0$ has an isolated root $z=z^{*}$, i.e., condition (2) is satisfied. From equation (12) as well as [12], it can be derived that functions $\mu, \vartheta$ and their partial derivative up to the second order are bounded for $z-z^{*}$ i.e., condition (3) is satisfied. The $i$ th subsystem of degraded system $\dot{p}=\mu\left(p, z^{*}, \rho\right)$ can be simplified as $\dot{p}_{i}=$ $-\delta_{i}\left(\lambda\left(p_{i}-p_{\mathrm{t}}\right)+\dot{p}_{\mathrm{t}}\right)$. Letting $e_{i}=p_{i}-p_{\mathrm{t}}$, we can further have $e_{i}(t)=\exp \left(-\delta_{i} \lambda t\right) e_{i}(0)$, with $e_{i}(0)$ denoting the initial position distance between $p_{i}$ and $p_{\mathrm{t}}$. Therefore, equilibrium point $\left(p^{*}, z^{*}\right)$ of system $\dot{p}=\mu(p, z, \rho)$ is exponentially stable, i.e., condition (4) is satisfied. In addition, with $\widehat{\tau}=t \rho$, the origin (i.e., $z-z^{*}=0$ ) of the bounded-layer system for system (12) can be formulated as

$$
\frac{\mathrm{d}\left(z-z^{*}\right)}{\mathrm{d} \widehat{\tau}}=\frac{\mathrm{d}(z)}{\mathrm{d} \widehat{\tau}}-0=\frac{1}{\rho} \dot{z},
$$

which is exponentially stable in view of Theorem 4 as well as Corollary 2 in [12] and condition (5) is thus satisfied.

Since all the five conditions are satisfied for all $t \in$ $(0,+\infty)$, according to Lemma 1 , there exist $1 / \rho^{*}>0$ (i.e., $\left.\rho^{*}>0\right)$ such that, for all $1 / \rho<1 / \rho^{*}$, i.e., for all $\rho>\rho^{*}$, system (12) exponentially converges to its equilibrium point.

Based on the above aspects (1) and (2), there exists $\rho^{*}>$ $0 \in \mathbb{R}$ such that, for all $\rho>\rho^{*}$, for a group of $n$ differentialdriven robots described by (5) with the coordination control law (12), $k$ robots with the minimum distance move towards the target at an exponential speed. The proof is thus complete.

\section{Distributed Competitive Tracking with Limited Communications}

We have developed centralized model (12) for competitive target tracking in the previous section, which requires allto-all communications. Aiming at reducing communication burdens, in this section, we develop a fully distributed model, which only requires limited communications.

Equation (11) indicates that only $\sum_{i=1}^{n} \sigma_{\Omega i}\left(z+v_{i} /(2 a)\right)$ requires the global information in the team, which can be deemed as the obstacle to achieving the goal of distributed coordination control. We use consensus filter to estimate it in a distributed manner. With consensus filter, a robot is able to estimate the average of filter inputs by running the following protocol [38]:

$$
\begin{aligned}
\dot{\zeta}_{i}= & -\gamma \sum_{j \in \mathbb{L}(i)} C_{i j}\left(\zeta_{i}-\zeta_{j}\right)-\gamma\left(\zeta_{i}-\sigma_{\Omega i}\left(z+\frac{v_{i}}{2 a}\right)\right) \\
& -\gamma \sum_{j \in \mathbb{L}(i)} C_{i j}\left(\varphi_{i}-\varphi_{j}\right), \\
\dot{\varphi}_{i}= & \sum_{j \in \mathbb{L}(i)} C_{i j}\left(\zeta_{i}-\zeta_{j}\right),
\end{aligned}
$$

where $\mathbb{L}(i)$ is the communication neighbor set of the $i$ th mobile robot; $\zeta_{i}$ and $\varphi_{i}$ are maintained by the $i$ th mobile robot; $C_{i j}>0$ for $j \in \mathbb{L}(i)$ with $C_{i j}=C_{j i} ; \gamma>0$ is used to scale the convergence rate. In addition, for $j \notin \mathbb{L}(i), C_{i j}=C_{j i}=0$. By running (17) on all the mobile robots involved, $\zeta_{i}$ converges to the average of inputs, i.e., $\sum_{i=1}^{n} \delta_{i} / n$ or $\sum_{i=1}^{n} \sigma_{\Omega i}\left(z+v_{i} /(2 a)\right) / n$.

Thus, by using consensus filter (17) to estimate $\sum_{i=1}^{n} \sigma_{\Omega i}\left(z+v_{i} /(2 a)\right)$, we further have

$$
\begin{aligned}
\dot{\zeta}_{i}= & -\gamma \sum_{j \in \mathbb{L}(i)} C_{i j}\left(\zeta_{i}-\zeta_{j}\right)-\gamma\left(\zeta_{i}-\sigma_{\Omega i}\left(z+\frac{v_{i}}{2 a}\right)\right) \\
& -\gamma \sum_{j \in \mathbb{L}(i)} C_{i j}\left(\varphi_{i}-\varphi_{j}\right),
\end{aligned}
$$




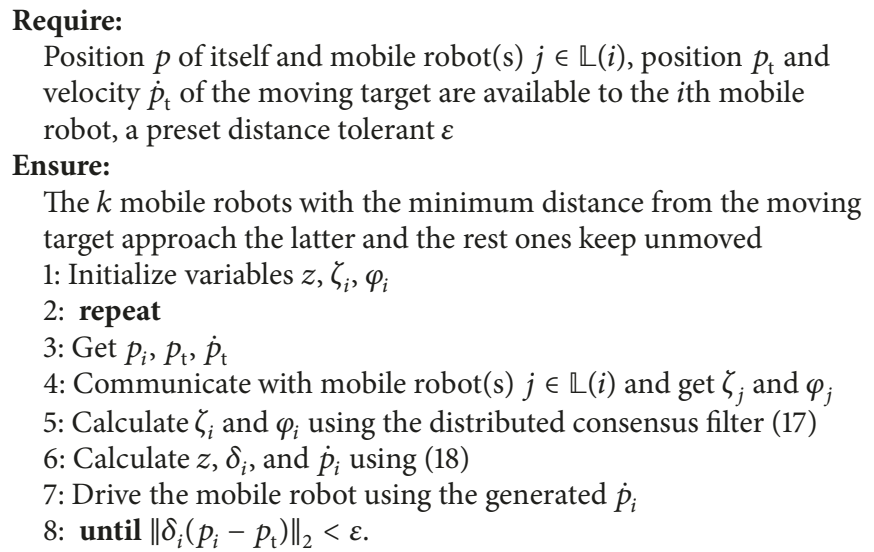

Algorithm 2: Distributed competitive target tracking control for the $i$ th mobile robot with limited communication.

$$
\begin{aligned}
\dot{\varphi}_{i} & =\sum_{j \in \mathbb{L}(i)} C_{i j}\left(\zeta_{i}-\zeta_{j}\right) \\
\dot{p}_{i} & =-\sigma_{\Omega i}\left(z+\frac{v_{i}}{2 a}\right)\left(\lambda\left(p_{i}-p_{\mathrm{t}}\right)+\dot{p}_{\mathrm{t}}\right), \\
\dot{z} & =-\rho\left(n \zeta_{i}-k\right),
\end{aligned}
$$

which can be written into a compact form:

$$
\begin{aligned}
& \dot{\zeta}=-\gamma L \zeta-\gamma(\zeta-w)-\gamma L \int_{t_{0}}^{t} L \zeta \mathrm{d} t, \\
& \dot{p} \\
& \quad=-\sigma_{\Omega}\left(z I_{2 n}+\frac{v}{2 a} \otimes I_{2}\right)\left(\lambda\left(p-I_{n} \otimes p_{\mathrm{t}}\right)+I_{n} \otimes \dot{p}_{\mathrm{t}}\right), \\
& \dot{z}=-\rho\left(I_{n}^{\mathrm{T}} \zeta-k\right),
\end{aligned}
$$

where $t_{0}$ denotes the initial time instant; $L=\operatorname{diag}\left(C I_{n}\right)-C$ with $\operatorname{diag}\left(C_{n}\right)$ being a diagonal matrix.

As illustrated in Figure 3, the proposed distributed coordination model consists of three parts: the $k$-WTA part and the distributed consensus filter part (as shown in the dashed rectangle in the figure) and the controller part. In addition to the information from the moving target and the $i$ th mobile robot itself, this model also requires $\zeta_{j}$ and $\varphi_{j}$, which originate from the distributed consensus filter part of mobile $\operatorname{robot}(s) j \in \mathbb{L}(i)$. Moreover, the procedures of the proposed distributed coordination model with limited communications is stated in Algorithm 2. The difference of Algorithms 1 and 2 lies in that a consensus filter is used to estimate $\delta_{i}$ in a distributed way in Algorithm 2. Distributed coordination control law (19) can be deemed as a cascaded system, where parameter $\rho$ is the gain of the outer loop (i.e., the $k$-WTA neural network model) to output the winners while $\gamma$ scales the convergence rate of the inner loop (i.e., the consensus filter) for estimating $\sum_{i=1}^{n} \delta_{i} / n$. Intuitively, it is necessary to choose the value of $\gamma$ larger than the value of $\rho$

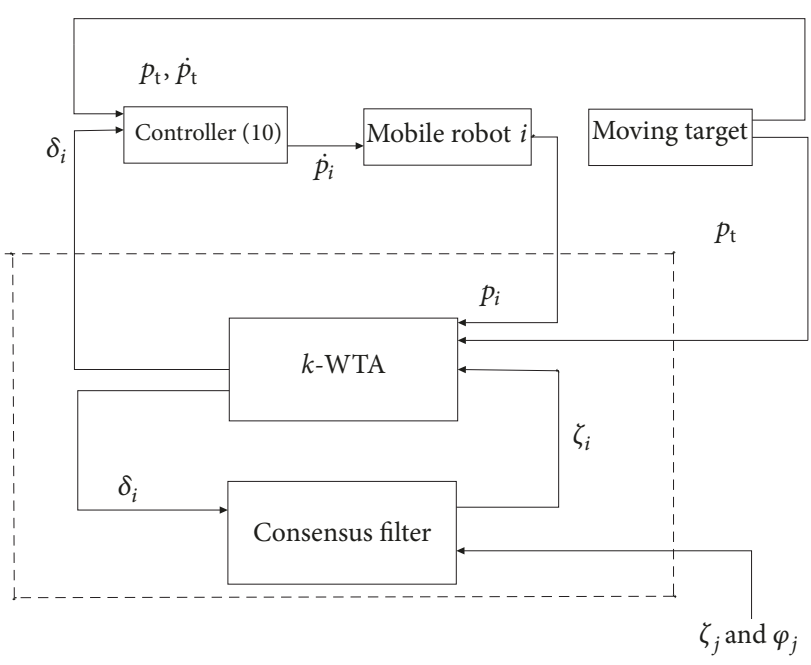

FIGURE 3: Control block diagram for the ith mobile robot in the distributed competitive target tracking control via multiple robots in the situation of limited communications, where subscript $j \in \mathbb{L}(i)$.

such that the inner loop converges much faster to obtain a stable system. We have the following theorem to reveal such a conclusion.

Theorem 4. There exists $\gamma^{*}>0 \in \mathbb{R}$ and $\rho^{*}>0 \in \mathbb{R}$ such that, for all $\gamma>\gamma^{*}>\rho>\rho^{*}$, for a group of $n$ differentialdriven robots described by (5) with the distributed coordination control law (19), $k$ robots with the minimum distance move towards the target at an exponential speed.

Proof. The proof involves two aspects. (1) The equilibrium point of system (12) is identical to the solution of Problem 2. (2) System (12) exponentially converges to its equilibrium point.

For aspect (1), it can be obtained from the proof of Theorem 3 and is thus omitted here. 
For aspect (2), the distributed coordination model (19) can be rewritten as

$$
\begin{aligned}
\dot{p} & =\mu(p, \beta, \rho, \gamma) \\
\frac{1}{\rho} \dot{\beta} & =\vartheta(p, \beta, \rho, \gamma),
\end{aligned}
$$

of which the equilibrium point is $\left(p^{*}, \beta^{*}\right)$, with $\dot{\beta}=$ $[\dot{z}, \dot{\zeta} \rho / \gamma]^{\mathrm{T}}$. Evidently, system (20) and its subsystem shown as follows are special cases of singularly perturbed system (1):

$$
\frac{1}{\rho} \dot{\beta}=\left[\begin{array}{c}
\dot{z} \\
\frac{\rho}{\gamma} \dot{\zeta}
\end{array}\right]=\left[\begin{array}{l}
\eta(z, \zeta, \rho, \gamma) \\
\theta(z, \zeta, \rho, \gamma)
\end{array}\right],
$$

of which the equilibrium point is $\left(z^{*}, \zeta^{*}\right)$. Then, the proof is divided into the following two steps: (1) the stability and convergence of subsystem (21) is evaluated via Lemma $1 ;(2)$ the stability and convergence of system (20) is evaluated via Lemma 1 based on theoretical results in Step 1.

Step 1 (the stability and convergence of subsystem (21)). Using the linear translation $z^{\prime}=z-z^{*}$ and $\zeta^{\prime}=\zeta-\zeta^{*}$, we have $z=z^{\prime}+z^{*}$ and $\zeta=\zeta^{\prime}+\zeta^{*}$, and further have

$$
\begin{aligned}
\dot{z}^{\prime} & =\eta\left(z^{\prime}+z^{*}, \zeta^{\prime}+\zeta^{*}, \rho, \gamma\right), \\
\frac{\rho}{\gamma} \dot{\zeta}^{\prime} & =\theta\left(z^{\prime}+z^{*}, \zeta^{\prime}+\zeta^{*}, \rho, \gamma\right) .
\end{aligned}
$$

It can be further derived that

$$
\begin{gathered}
\dot{z}^{\prime}=\eta\left(z^{*}, \zeta^{*}, \rho, \gamma\right)=0, \\
\frac{\rho}{\gamma} \dot{\zeta}^{\prime}=\theta\left(z^{*}, \zeta^{*}, \rho, \gamma\right)=0,
\end{gathered}
$$

where $z^{\prime}=\zeta^{\prime}=0$ are the new equilibrium points.

Therefore, condition (1) is satisfied. It can be generalized from [38] that $L \zeta+(\zeta-w)+L \int_{t_{0}}^{t} L \zeta \mathrm{d} t=0$ has an isolated $\operatorname{root} \zeta=\zeta^{*}$, i.e., condition (2) is satisfied. From equation (21), it can be derived that functions $z, \zeta$ and their partial derivative up to the second order are bounded for $\zeta-\zeta^{*}$ i.e., condition (3) is satisfied. The degraded system $\dot{z}=\eta\left(\zeta, z^{*}, \rho, \gamma\right)$ can be formulated as $\dot{z}=-\rho\left(I_{n}^{\mathrm{T}} \zeta^{*}-k\right)=0$. Therefore, equilibrium point $\left(z^{*}, \zeta^{*}\right)$ of system $\dot{z}=\eta(z, \zeta, \rho, \gamma)$ is exponentially stable, i.e., condition (4) is satisfied. In addition, with $\widehat{\tau}=$ $t \gamma / \rho$, the origin (i.e., $\zeta-\zeta^{*}=0$ ) of the bounded-layer system for subsystem (21) can be formulated as

$$
\frac{\mathrm{d}\left(\zeta-\zeta^{*}\right)}{\mathrm{d} \widehat{\tau}}=\frac{\mathrm{d}(\zeta)}{\mathrm{d} \hat{\tau}}-0=\frac{\rho}{\gamma} \dot{\zeta},
$$

which is exponentially stable in view of Theorem 5 in [38] and condition (5) is thus satisfied.

Since all the five conditions are satisfied for all $t \in$ $(0,+\infty)$, according to Lemma 1 , there exist $\rho^{*} / \gamma^{*}>0$ (i.e., $\gamma^{*}>\rho^{*}>0$ ) such that, for all $\gamma>\gamma^{*}>\rho>\rho^{*}$, the equilibrium point $\left(z^{*}, \zeta^{*}\right)$ of subsystem (21) is exponentially stable. The proof of Step 1 is thus complete.
Step 2 (the stability and convergence of system (20)). By following the similar procedures as those in Theorem 3 and the above Step 1 and based on the theoretical results therein, one can derive the conclusion that there exists $\gamma^{*}>0 \in \mathbb{R}$ and $\rho^{*}>0 \in \mathbb{R}$ such that, for all $\gamma>\gamma^{*}>\rho>\rho^{*}$, the equilibrium of system (19) is exponentially stable.

Based on the above aspects (1) and (2), there exists $\gamma^{*}>$ $0 \in \mathbb{R}$ and $\rho^{*}>0 \in \mathbb{R}$ such that, for all $\gamma>\gamma^{*}>\rho>\rho^{*}$, for a group of $n$ differential-driven robots described by (5) with the distributed coordination control law (19), $k$ robots with the minimum distance move towards the target at an exponential speed. The proof is thus complete.

For the distributed coordination model (19) for competitive target tracking via multiple mobile robots, the core idea is to use the distributed consensus filter to estimate the centralized quantity in Algorithm 1 and use the estimated value to replace the actual centralized quantity in the moving target tracking. Theoretical analysis of the convergence and stability of distributed coordination model (19) are presented in Theorem 4, which reveals the key point for a successful competitive target tracking is that the consensus filter should run on a fast enough time scale.

\section{Illustrative Examples}

In this section, computer simulations are provided based on ten differential-driven-wheeled mobile robots. In what follows, the parameters are set as $a=0.1, \lambda=10, \rho=10^{5}$, $\gamma=10^{5}, \varepsilon=0.01 \mathrm{~m}$ with the rest ones being set as 0 initially. In addition, $C_{i j}=1$ for $|i-j| \leq 1$; otherwise, $C_{i j}=0$.

Example 1. In this example, model (12) with all-to-all communications is employed to the task of moving target tracking with $k=2$. The corresponding simulation results, which are classified into two phases, are illustrated in Figures 4-6.

Specifically, as shown in Figure $4(\mathrm{a})$, at $t=0 \mathrm{~s}$, the initial position of the moving target is around $(1,9.5)$, from which the two nearest mobile robots marked in red lines win the competition. In comparison, the rest ones, as the losers of the competition, are deactivated and unmoved. As a result, the two winners begin to track the moving target. In addition, as visualized in Figure 4(b), at $t=1 \mathrm{~s}$, the target moves to the position around $(0.7,7)$, followed by the two winner mobile robots marked in red lines. It can be seen from this figure that the two winners approach to the moving target, which means that the tracking task goes well. Moreover, Figure 4(c) illustrates the snapshot at $t=$ $3 \mathrm{~s}$, from which, we can find that the two winner mobile robots have almost achieved the position of the moving target. Besides, the distance tolerant $\varepsilon=0.01 \mathrm{~m}$ means that, during the competitive target tracking process, for the first time that the norm of the distance between a mobile robot and the target is less than $0.01 \mathrm{~m}$, the moving target can be deemed as captured by the mobile robot. The corresponding actual path of the moving target as well as the tracking trajectories are shown in Figure 4(d), from which, we can 


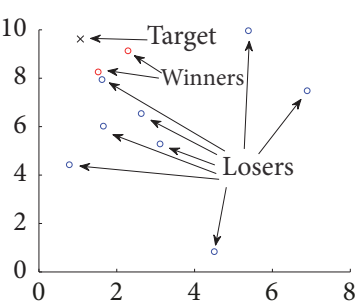

(a)

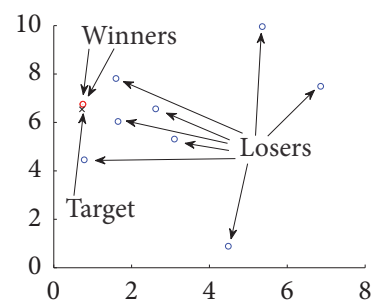

(b)

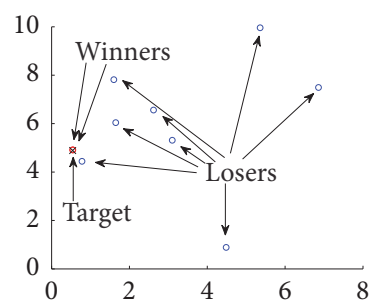

(c)

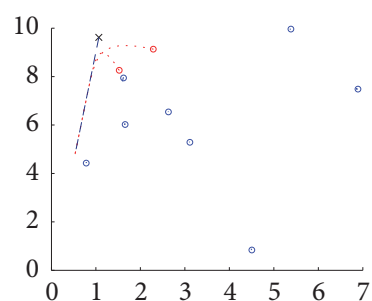

(d)

Figure 4: (Phase 1) With $k=2$, snapshots for centralized model (12) with all-to-all communications for moving target tracking and the corresponding tracking trajectories, where initial locations of mobile robots and the moving target are randomly generated. In this sense, ten mobile robots compete with each other for tracking the moving target, and only two of them with the minimum distance from the moving target (the winners) are activated to track while the rest ones (the losers) keep unmoved. (a) Snapshots at $t=0 \mathrm{~s}$. (b) Snapshots at $t=1 \mathrm{~s}$. (c) Snapshots at $t=3 \mathrm{~s}$. (d) Actual path of the moving target and tracking trajectories of different mobile robots.

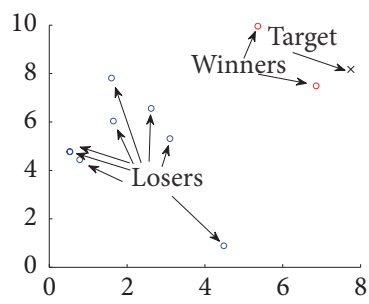

(a)

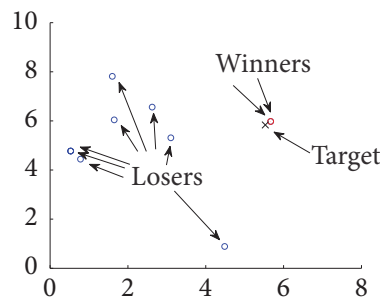

(b)

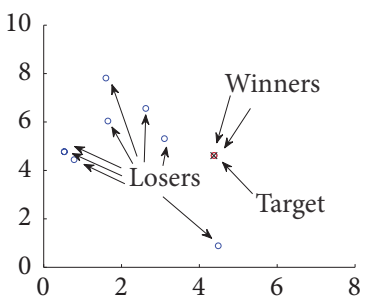

(c)

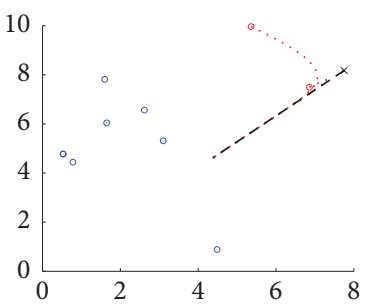

(d)

Figure 5: (Phase 2) As the continuation of simulation results shown in Figure 4, this figure shows the tracking trajectory and snapshots for centralized model (12) with all-to-all communications for moving target tracking and initial locations of mobile robots being the same as those in Figure 4(c) and the initial location of moving target being generated randomly. Ten mobile robots compete with each other for tracking the moving target, and only two of them with the minimum distance from the moving target (the winners) are activated to track while the rest ones (the losers) keep unmoved. (a) The snapshot at $t \approx 4 \mathrm{~s}$. (b) The snapshot at $t=5 \mathrm{~s}$. (c) The snapshot at $t=8 \mathrm{~s}$. (d) Tracking trajectories of different mobile robots.

find that the trajectories of the two winner mobile robots are smooth. In addition, as the losers in the sense of a further distance from the target, the rest mobile robots keep unmoved, which can be deemed as a measure of energy conservation. These simulation results reveal preliminarily the effectiveness of the proposed coordination model (12) with all-to-all communications.

As the continuation of simulation results shown in Figure 4 , the simulation results on phase 2 of the target tracking are shown in Figure 5. Initial locations $(t \approx 4$ s) of all mobile robots of phase 2 are the same as their finial locations of phase 1 shown in Figure 4(c) and the moving target is randomly placed. At the beginning of phase 2, as illustrated in Figure 5(a), the moving target appears at the location $(7.5,8.5)$, and the mobile robots marked in red win the competition and are activated. In addition, as visualized in Figure 5(b), at $t=5 \mathrm{~s}$, the target moves to the position $(5.5,5.5)$ and the two winners are very close to the moving target. Moreover, Figure 4(c) illustrates the snapshot at $t=8 \mathrm{~s}$, where the locations of the two mobile robots (the winners) and the target are nearly at the same position. To see the overall target tracking process more clearly, Figure 5(d) visualizes the actual path of the moving target as well as the tracking trajectories. From the figure, we can find that the trajectories of the two mobile robots (the winners) are smooth and the rest mobile robots keep unmoved. These simulation results verify the effectiveness of the proposed coordination model (12) with all-to-all communications.

To observe the target tracking task in a different perspective, outputs of the $k$-WTA network of the whole process as well as the corresponding velocities of mobile robots at different phases are illustrated in Figure 6. It can be found in Figure 6(a) that, starting with randomly generated initial state, the outputs of the $k$-WTA network rapidly converge to the correct results within $8 \times 10^{-4} \mathrm{~s}$, and the outputs change rapidly when the target tracking process enters into phase 2 . The detailed velocities of the mobile robots in phase 1 and phase 2 are shown in Figures 6(b) and 6(c), respectively. From these two subfigures, it can be observed that the velocities of two mobile robots eventually reach to zero while the rest ones' velocities stay at zero during each phase. These results further verify the effectiveness of the proposed coordination model (12) with all-to-all communications in a different perspective.

Example 2. In this example, the distributed coordination model (19) with limited communications is employed to track the moving target with a single winner, i..e, $k=1$. As stated above, we assume that the mobile robots with $\mid i-$ $j \mid \leq 1$ have the communications and the rest ones can not communicate with each other for information exchanging. The corresponding simulation results, which are classified into two phases, are illustrated in Figures 7-9. 


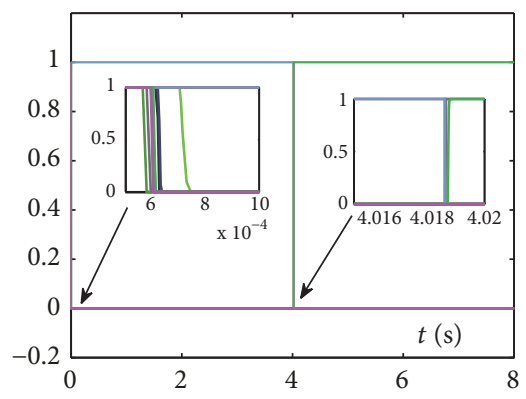

(a)

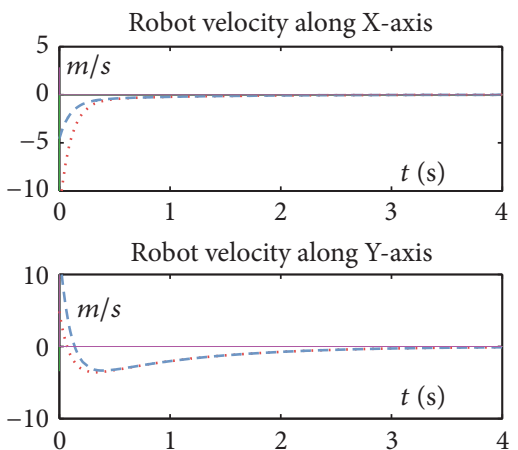

(b)

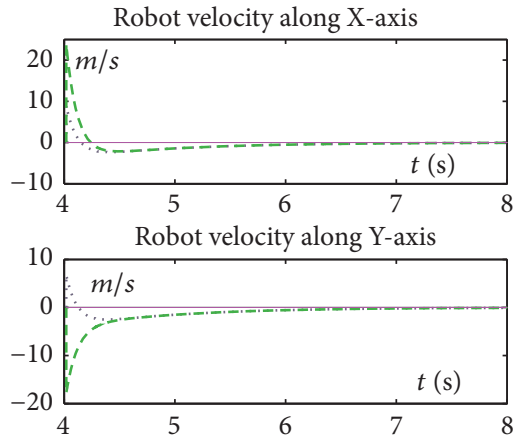

(c)

FIGURE 6: Outputs of $k$-WTA network in centralized model (12) with $k=2$ as well as the corresponding velocities of mobile robots at different phases. (a) Outputs of $k$-WTA network with $k=2$. (b) Velocities of mobile robots of phase 1. (c) Velocities of mobile robots of phase 2.

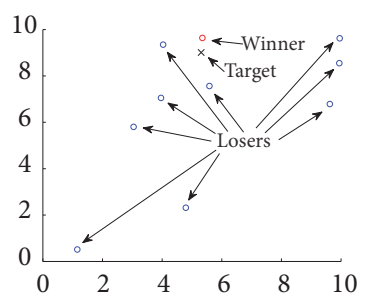

(a)

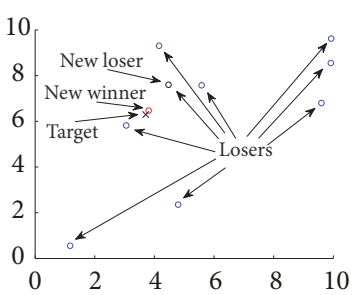

(b)

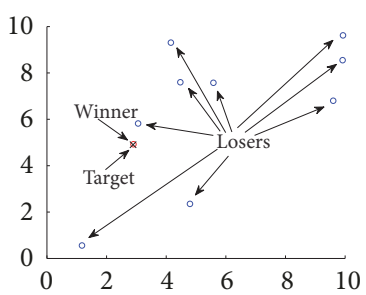

(c)

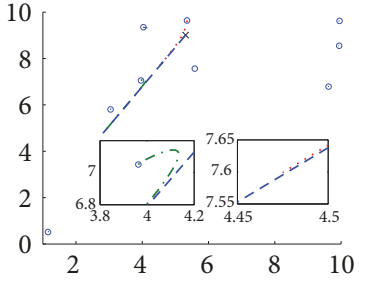

(d)

Figure 7: (Phase 1) With $k=1$, snapshots for distributed coordination model (19) with limited communications for tracking the moving target and the corresponding tracking trajectories, where initial locations of mobile robots and the moving target are randomly generated. In this sense, at each time instant, ten mobile robots compete with each other for tracking the moving target, and only one of them with the minimum distance from the moving target (the winner) is activated to track while the rest ones (the losers) keep unmoved. At $t \approx 0.5 \mathrm{~s}$, as the moving target approaches to one of the losers, the winner at the initial time fails in the competition and becomes a loser marked in black line. As a continuator, the new winner marked in red line begins to track the moving target. (a) Snapshots at $t=0 \mathrm{~s}$. (b) Snapshots at $t=1 \mathrm{~s}$. (c) Snapshots at $t=3 \mathrm{~s}$. (d) Tracking trajectories of different mobile robots.

Specifically, as shown in Figure 7(a), at $t=0 \mathrm{~s}$, the initial position of the moving target is $(5.5,9)$, from which the nearest mobile robot at $(5.5,9.5)$ marked in red lines wins the competition. In comparison, the rest ones, as the losers of the competition, are deactivated and unmoved. As a result, the winner begins to track the moving target. However, as shown in Figure 7(b), as the moving target approaches to one of the losers, the incipient winner loses out at $t \approx$ $0.5 \mathrm{~s}$ (thus is marked in black line in Figure 7(b)). As a result, the new winner marked in red line begins to track the moving target. Moreover, Figure 7(c) illustrates the snapshot at $t=3 \mathrm{~s}$, from which, it can be observed that the mobile robot (the new winner) has almost approached the moving target. Figure 7(d) illustrates the corresponding actual path of the moving target as well as the tracking trajectories, which shows that the trajectories of the two mobile robots (the new winner and the new loser) are smooth. In addition, as the losers, the rest mobile robots keep unmoved. These simulation results verify preliminarily the effectiveness of the proposed distributed coordination model (19) with limited communications.

Simulation results on phase 2 of the target tracking are shown in Figure 8, which is the continuation of the simulation results shown in Figure 7. Initial locations $(t \approx 4 \mathrm{~s})$ of all mobile robots of phase 2 are the same as their final locations of phase 1 shown in Figure 7(c) and the moving target is randomly placed initially. At the beginning of phase 2 , as illustrated in Figure 8(a), the moving target is randomly placed at $(4.5,5.5)$, and the mobile robot marked in red wins the competition and is activated to track the moving target. In addition, Figure 8(b) visualizes that, at $t=5$ $\mathrm{s}$, the target moves to the position around $(2.5,3)$ and the winner is close to the moving target. Moreover, as illustrated in Figure 8(c), the mobile robot (the winner) finally captures the moving target and the task is completed successfully. Figure 8(d) visualizes the actual path of the moving target as well as the tracking trajectories, from which, we can find that the trajectory of the winner is smooth and the rest mobile robots keep unmoved. These simulation results verify the effectiveness of the proposed distributed coordination model (19) with limited communications.

Figure 9 provides a different perspective to observe the target tracking task, which illustrates outputs of the $k$-WTA network of the whole process as well as the corresponding velocities of mobile robots at different phases. It can be found in Figure 9(a) that the outputs of the $k$-WTA network rapidly converge to the correct results within $0.1 \mathrm{~s}$, which is evidently longer than that shown in Figure 6(a) due to the estimation 


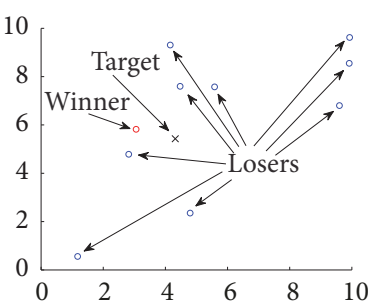

(a)

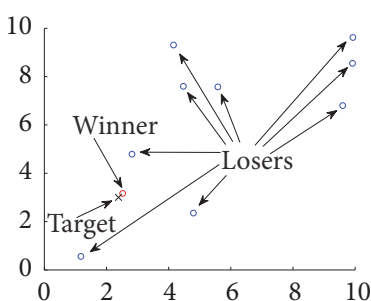

(b)

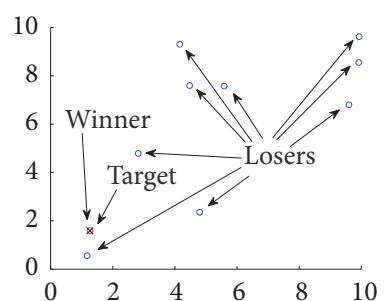

(c)

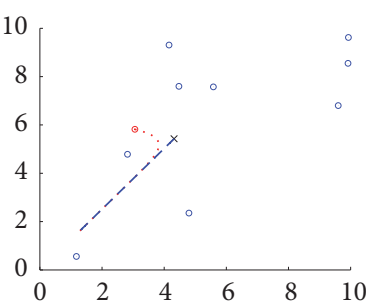

(d)

Figure 8: (Phase 2) As the continuation of simulation results shown in Figure 7, this figure shows the tracking trajectory and snapshots for the distributed coordination model (19) with limited communications for tracking the moving target and initial locations of mobile robots being the same as those in Figure 7(c) and the initial location of moving target being generated randomly. Ten mobile robots compete with each other for tracking the moving target, and only the one with the minimum distance from the moving target is activated to track while the rest ones (the losers) keep unmoved. (a) The snapshot at $t \approx 4.1 \mathrm{~s}$. (b) The snapshot at $t=5 \mathrm{~s}$. (c) The snapshot at $t=8 \mathrm{~s}$. (d) Tracking trajectories of different mobile robots.

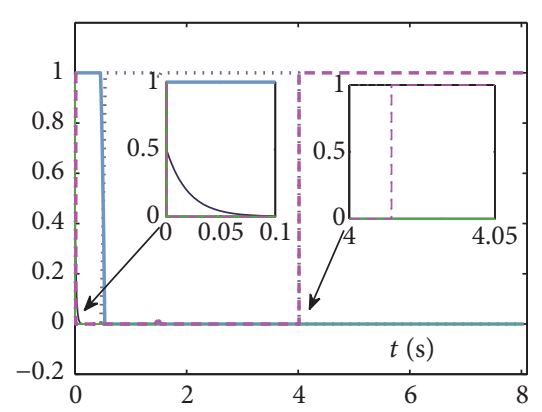

(a)

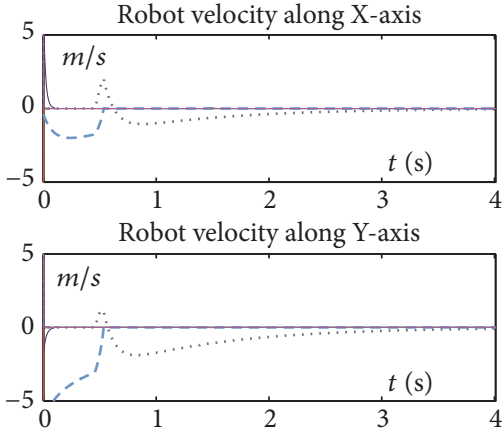

(b)

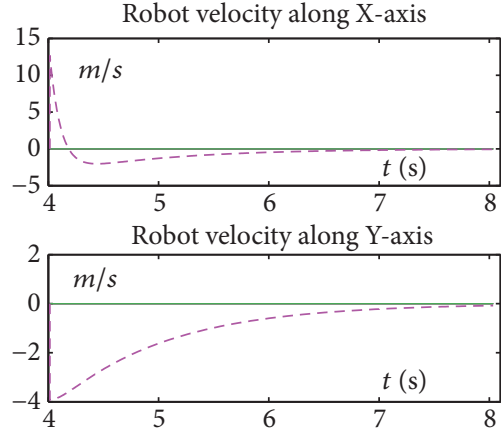

(c)

FIGURE 9: Outputs of $k$-WTA network in distributed coordination model (19) with $k=1$ as well as the corresponding velocities of mobile robots at different phases. (a) Outputs of $k$-WTA network with $k=2$. (b) Velocities of mobile robots of phase 1. (c) Velocities of mobile robots of phase 2 .

on $\sum_{i=1}^{n} g_{\Omega i}\left(z+v_{i} /(2 a)\right)$ via consensus filter (17). The velocities of the mobile robots in phase 1 and phase 2 are shown in Figures 9(b) and 9(c), respectively. From these two subfigures, it can be observed that the velocities of two winner mobile robots eventually reach to zero while the rest ones' velocities remain zero during each phase. These results further verify the effectiveness of the proposed distributed coordination model (19) with limited communications.

Example 3. Simulation results with zero-initial-velocity constraint, as well as bound limits on velocity of mobile robots are shown in Figure 10, with $\dot{p}_{i}^{+}=-\dot{p}_{i}^{-}=3 \mathrm{~m} / \mathrm{s}$. Specifically, as shown in Figure 10(a), starting with randomly generated initial state, the outputs of the $k$-WTA network rapidly converge to the correct results. The detailed velocities of the mobile robots are shown in Figure 10(b). It can be observed that the velocities of two mobile robots eventually reach to zero while the rest ones' velocities stay at zero. In addition, the velocities are kept within their physical limits as well. Moreover, each winner mobile robot, starting from zero velocity, gradually accelerates its speed to reach the desired velocity. The corresponding actual path of the moving target as well as the tracking trajectories are shown in Figure 10(c), from which, we can find that the trajectories of the two winner mobile robots are smooth. In addition, as the losers in the sense of a further distance from the target, the rest mobile robots keep unmoved, which can be deemed as a measure of energy conservation.

\section{Conclusions}

In this paper, we have proposed centralized and distributed coordination models with all-to-all and limited communications, respectively. Simulations based on differential-drivenwheeled mobile robots have been conducted to illustrate the efficacy of the proposed centralized and distributed coordination models for tracking moving target in a competition manner with all-to-all communications and limited communications.

\section{Data Availability}

The data used to support the findings of this study are available from the corresponding author upon request. 


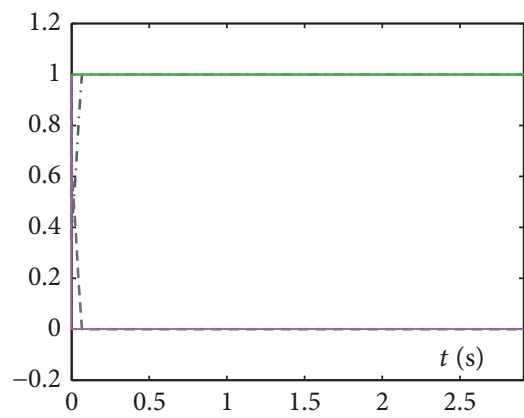

(a)
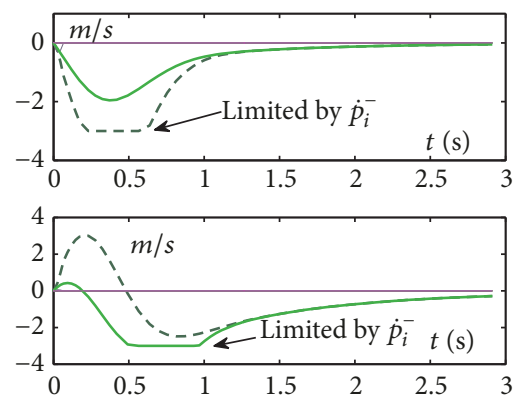

(b)

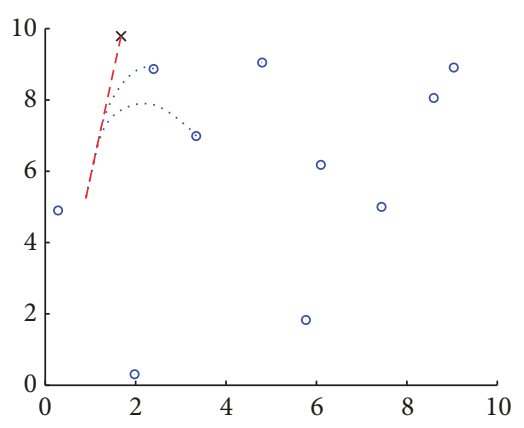

(c)

FIGURE 10: Performance of $k$-WTA network in distributed coordination model with $k=2$, zero-initial-velocity constraint, as well as bound limits on velocity of mobile robots. (a) Outputs of $k$-WTA network with $k=2$. (b) Velocities of mobile robots. (c) Tracking trajectories of different mobile robots.

\section{Conflicts of Interest}

The authors declare that there are no conflicts of interest regarding the publication of this paper.

\section{Acknowledgments}

This work is supported by the National Natural Science Foundation of China (no. 61703189), by the Fund of Key Laboratory of IoT of Qinghai Province (no. 2017-ZJ-Y21), by the Natural Science Foundation of Gansu Province, China (no. 18JR3RA264), by the Guangdong Outstanding Young Teacher Training Program in Higher School (no. YQ2015104), by the Guangzhou Higher School Innovation and Entrepreneurship Education Project (2017153201), by the Opening Foundation of Key Laboratory of Opto-Technology and Intelligent Control, Ministry of Education (no. KFKT2018-1), by the National Natural Science Foundation of China (no. 11561029), by the Belt and Road Special Project of Lanzhou University (no. 2018ldbryb020), by the Fundamental Research Funds for the Central Universities (no. lzujbky-2017-37), by the Fund of Robot Technology Used for Special Environment Key Laboratory of Sichuan Province (no. 17kfkt03), by the Natural Science Foundation of Hunan Province (no. 2017JJ3257), by the Research Foundation of Education Bureau of Hunan Province, China (no. 17C1299), by Hong Kong Research Grants Council Early Career Scheme (with no. 25214015), by Hong Kong Polytechnic University (with nos. G-YBMU, G-UA7L, 4-ZZHD, F-PP2C, and 4-BCCS), and also by the outstanding Youth Foundation of Shandong Province (ZR2016JL024).

\section{References}

[1] L. Jin, S. Li, X. Luo, Y. Li, and B. Qin, "Neural Dynamics for Cooperative Control of Redundant Robot Manipulators," IEEE Transactions on Industrial Informatics, vol. 14, no. 9, pp. 38123821, 2018.

[2] L. Cheng, Y. Wang, W. Ren, Z.-G. Hou, and M. Tan, "Containment Control of Multiagent Systems with Dynamic Leaders Based on a PIn-Type Approach," IEEE Transactions on Cybernetics, vol. 46, no. 12, pp. 3004-3017, 2016.
[3] C. L. P. Chen, G.-X. Wen, Y.-J. Liu, and F.-Y. Wang, "Adaptive consensus control for a class of nonlinear multiagent time-delay systems using neural networks," IEEE Transactions on Neural Networks and Learning Systems, vol. 25, no. 6, pp. 1217-1226, 2014.

[4] L. Jin, S. Li, B. Hu, and C. Yi, "Dynamic neural networks aided distributed cooperative control of manipulators capable of different performance indices," Neurocomputing, vol. 291, pp. 50-58, 2018.

[5] K. M. Lynch, I. B. Schwartz, P. Yang, and R. A. Freeman, "Decentralized environmental modeling by mobile sensor networks," IEEE Transactions on Robotics, vol. 24, no. 3, pp. 710-724, 2008.

[6] S. Li, M. Zhou, X. Luo, and Z.-H. You, "Distributed winnertake-all in dynamic networks," Institute of Electrical and Electronics Engineers Transactions on Automatic Control, vol. 62, no. 2, pp. 577-589, 2017.

[7] X. Li and S. Song, "Stabilization of Delay Systems: delaydependent Impulsive Control," IEEE Transactions on Automatic Control, vol. 62, no. 1, pp. 406-411, 2017.

[8] L. Jin and S. Li, "Distributed Task Allocation of Multiple Robots: A Control Perspective," IEEE Transactions on Systems, Man, and Cybernetics: Systems, pp. 1-9.

[9] W. Maass, "On the computational power of winner-take-all," Neural Computation, vol. 12, no. 11, pp. 2519-2535, 2000.

[10] S. Liu and J. Wang, "A simplified dual neural network for quadratic programming with its KWTA application," IEEE Transactions on Neural Networks and Learning Systems, vol. 17, no. 6, pp. 1500-1510, 2006.

[11] Shubao L. and Jun W., "A new k-winners-take-all neural network," in Proceedings of the International Joint Conference on Neural Networks 2005, pp. 712-716, Montreal, Que., Canada.

[12] X. Hu and J. Wang, "An improved dual neural network for solving a class of quadratic programming problems and its k-winners-take-all application," IEEE Transactions on Neural Networks and Learning Systems, vol. 19, no. 12, pp. 2022-2031, 2008.

[13] S. Li, B. Liu, and Y. Li, "Selective positive-negative feedback produces the winner-take-all competition in recurrent neural networks," IEEE Transactions on Neural Networks and Learning Systems, vol. 24, no. 2, pp. 301-309, 2013.

[14] H. Wang, X. Liu, and K. Liu, "Robust adaptive neural tracking control for a class of stochastic nonlinear interconnected systems," IEEE Transactions on Neural Networks and Learning Systems, vol. 27, no. 3, pp. 510-523, 2016. 
[15] L. Jin, S. Li, B. Hu, M. Liu, and J. Yu, "Noise-Suppressing Neural Algorithm for Solving Time-Varying System of Linear Equations: A Control-Based Approach," IEEE Transactions on Industrial Informatics, pp. 1-1.

[16] Y. Zhang and J. Wang, "Obstacle avoidance for kinematically redundant manipulators using a dual neural network," IEEE Transactions on Systems, Man, and Cybernetics, Part B: Cybernetics, vol. 34, no. 1, pp. 752-759, 2004.

[17] Y. Huang, J. Na, X. Wu, X. Liu, and Y. Guo, "Adaptive control of nonlinear uncertain active suspension systems with prescribed performance," ISA Transactions $^{\circledR}$, vol. 54, pp. 145-155, 2015.

[18] Y.-J. Liu, S. Li, S. Tong, and C. L. Chen, "Neural approximationbased adaptive control for a class of nonlinear nonstrict feedback discrete-time systems," IEEE Transactions on Neural Networks and Learning Systems, vol. 28, no. 7, pp. 1531-1541, 2017.

[19] Y. Huang, J. Na, X. Wu, and G. Gao, "Approximation-Free Control for Vehicle Active Suspensions with Hydraulic Actuator," IEEE Transactions on Industrial Electronics, vol. 65, no. 9, pp. 7258-7267, 2018.

[20] X. Li and J. Wu, "Stability of nonlinear differential systems with state-dependent delayed impulses," Automatica, vol. 64, pp. 6369, 2016.

[21] C. Yang, C. Zeng, P. Liang, R. Li, and C. Su, "Interface design of a physical human $\cap$ crobot interaction system for human impedance adaptive skill transfer," IEEE Trans. Automat. Sci. Eng, vol. 15, no. 1, pp. 329-340, 2018.

[22] C. Yang, X. Wang, Z. Li, Y. Li, and C. Su, "Teleoperation control based on combination of wave variable and neural networks," IEEE Transactions on Systems, Man, and Cybernetics: Systems, 2017.

[23] C. Yang, Y. Jiang, Z. Li, W. He, and C.-Y. Su, "Neural control of bimanual robots with guaranteed global stability and motion precision," IEEE Transactions on Industrial Informatics, 2017.

[24] C. Yang, X. Wang, L. Cheng, and H. Ma, "Neural-learningbased telerobot control with guaranteed performance," IEEE Transactions on Cybernetics, 2017.

[25] L. Jin, S. Li, H. M. La, and X. Luo, "Manipulability optimization of redundant manipulators using dynamic neural networks," IEEE Transactions on Industrial Electronics, vol. 64, no. 6, pp. 4710-4720, 2017.

[26] T. Li, H. Zhao, and Y. Chang, "Delay-Dependent Stability in Uncalibrated Image-Based Dynamic Visual Servoing Robotic System," Complexity, vol. 2018, pp. 1-14, 2018.

[27] C. Yang, Y. Xu, L. Zhou, and Y. Sun, "Model-Free Composite Control of Flexible Manipulators Based on Adaptive Dynamic Programming," Complexity, vol. 2018, pp. 1-9, 2018.

[28] L. Jin, Y. Zhang, S. Li, and Y. Zhang, "Modified ZNN for TimeVarying Quadratic Programming with Inherent Tolerance to Noises and Its Application to Kinematic Redundancy Resolution of Robot Manipulators," IEEE Transactions on Industrial Electronics, vol. 63, no. 11, pp. 6978-6988, 2016.

[29] L. Jin and Y. Zhang, "G2-Type SRMPC scheme for synchronous manipulation of two redundant robot arms," IEEE Transactions on Cybernetics, vol. 45, no. 2, pp. 153-164, 2015.

[30] L. Jin, S. Li, L. Xiao, R. Lu, and B. Liao, "Cooperative Motion Generation in a Distributed Network of Redundant Robot Manipulators With Noises," IEEE Transactions on Systems, Man, and Cybernetics: Systems, vol. 48, no. 10, pp. 1715-1724, 2018.

[31] Z. Y. Wang and D. B. Gu, "Cooperative target tracking control of multiple robots," IEEE Transactions on Industrial Electronics, vol. 59, no. 8, pp. 3232-3240, 2012.
[32] L. Xiao, S. Li, F. Lin, Z. Tan, and A. H. Khan, "Zeroing Neural Dynamics for Control Design: Comprehensive Analysis on Stability, Robustness, and Convergence Speed," IEEE Transactions on Industrial Informatics, pp. 1-1.

[33] L. Xiao, B. Liao, S. Li, Z. Zhang, L. Ding, and L. Jin, "Design and Analysis of FTZNN Applied to the Real-Time Solution of a Nonstationary Lyapunov Equation and Tracking Control of a Wheeled Mobile Manipulator," IEEE Transactions on Industrial Informatics, vol. 14, no. 1, pp. 98-105, 2018.

[34] L. Jin, S. Li, H. La, X. Zhang, and B. Hu, "Dynamic task allocation in multi-robot coordination for moving target tracking: a distributed approach," Automatica, no. 99, 2018.

[35] N. Lashkari, M. Biglarbegian, and S. X. Yang, "Development of a new robust controller with velocity estimator for docked mobile robots: theory and experiments," IEEE/ASME Transactions on Mechatronics, vol. 22, no. 3, pp. 1287-1298, 2017.

[36] H. K. Khalil, Nonlinear Systems, Prentice-Hall, NJ, USA, 3nd edition, 2002.

[37] S. Li, R. Kong, and Y. Guo, "Cooperative distributed source seeking by multiple robots: Algorithms and experiments," IEEE/ASME Transactions on Mechatronics, vol. 19, no. 6, pp. 1810-1820, 2014.

[38] R. A. Freeman, P. Yang, and K. M. Lynch, "Stability and Convergence Properties of Dynamic Average Consensus Estimators," in Proceedings of the 45th IEEE Conference on Decision and Control, pp. 338-343, San Diego, CA, USA, December 2006. 


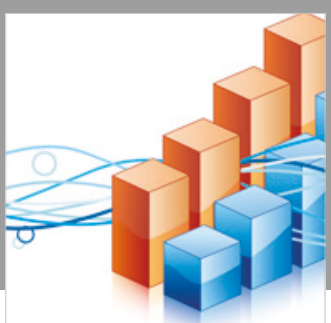

Advances in

Operations Research

\section{-n-m}
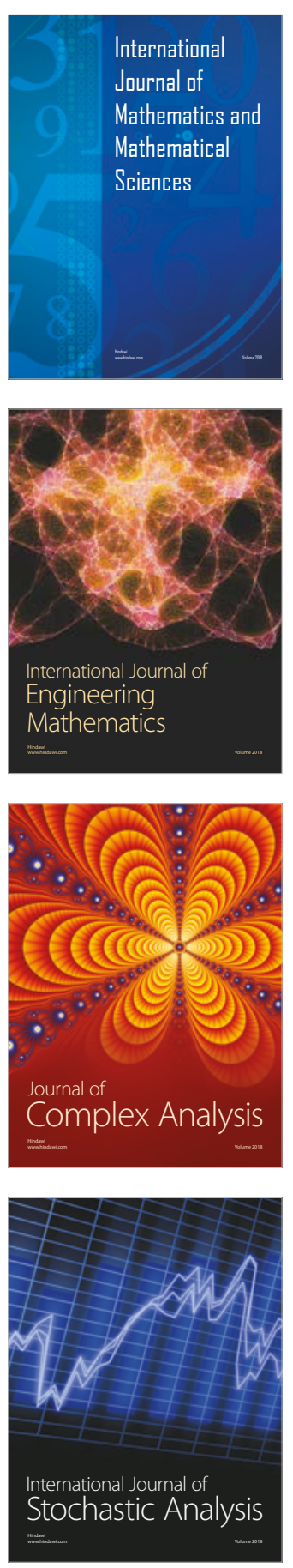
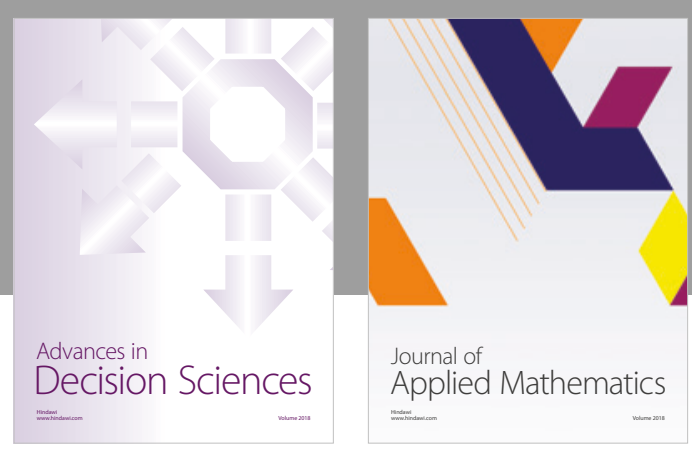

Journal of

Applied Mathematics
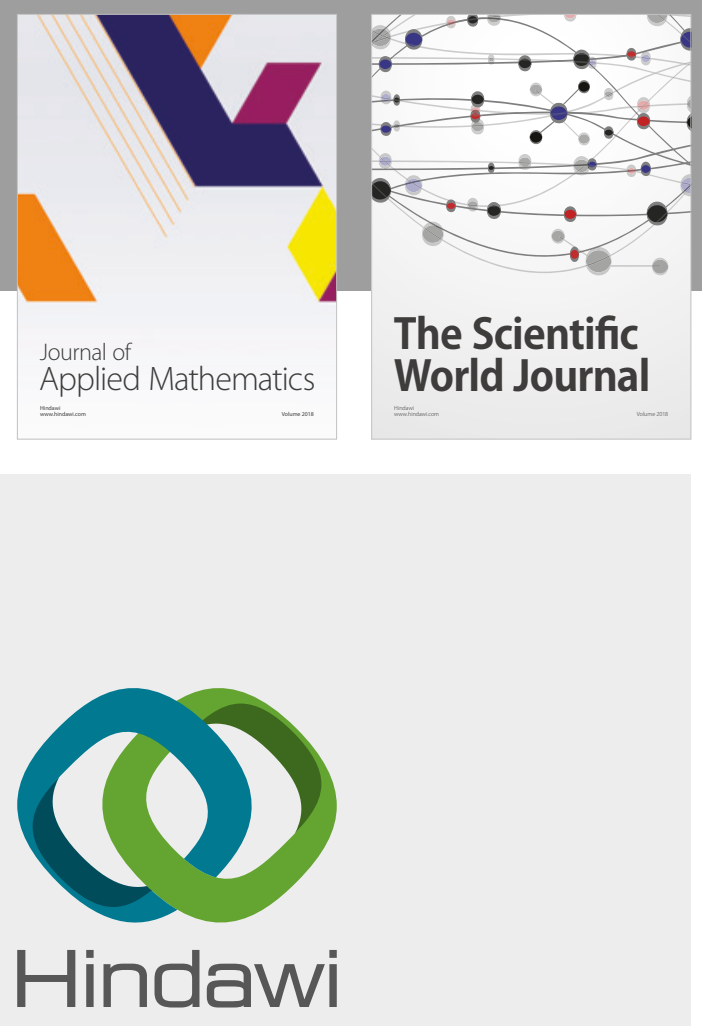

Submit your manuscripts at

www.hindawi.com

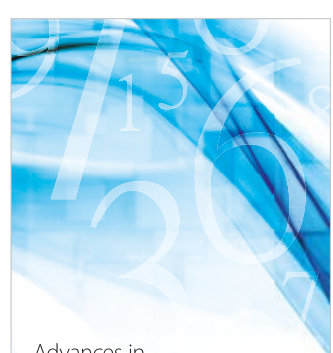

Advances in
Numerical Analysis
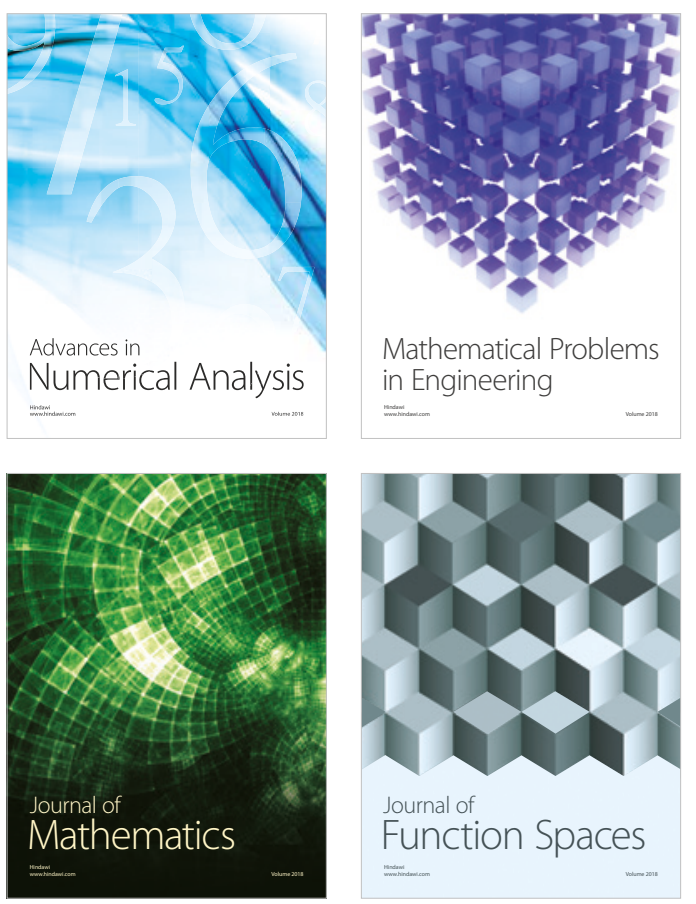

Mathematical Problems in Engineering

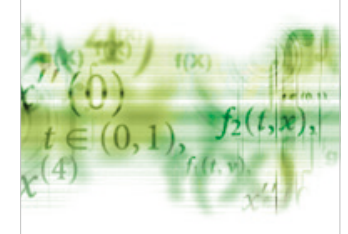

International Journal of

Differential Equations

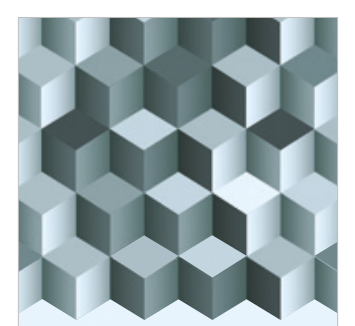

Journal of

Function Spaces
The Scientific

World Journal

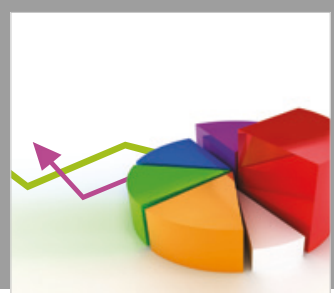

Journal of

Probability and Statistics
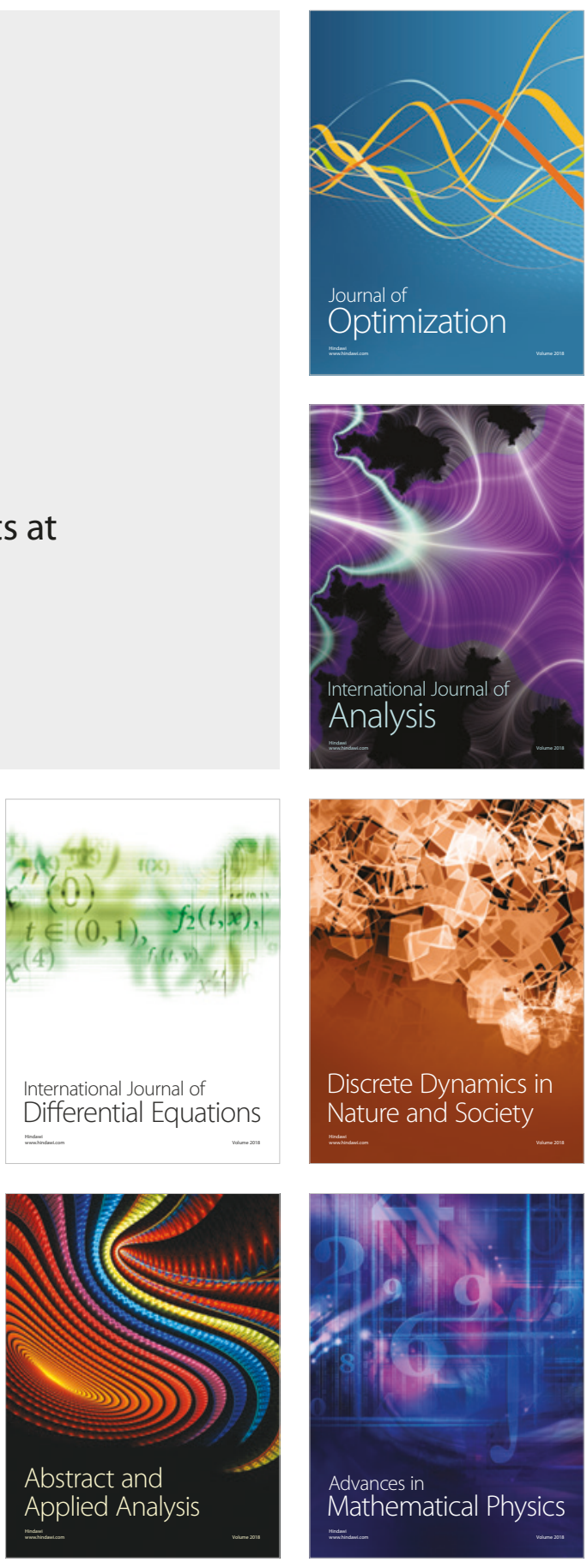